\title{
The Closer We Get, The Further Apart We Become
}

\author{
Julia Karady ${ }^{1}$, Pal Maurovich-Horvat ${ }^{2}$ \\ ${ }^{1}$ Cardiac MR PET CT Program, Massachusetts General Hospital, Harvard Medical School, Boston, MA, USA \\ ${ }^{2}$ MTA-SE Cardiovascular Imaging Research Group, Heart and Vascular Center, Semmelweis University, Budapest, Hungary
}

Coronary computed tomography angiography (CTA) became a powerful tool in the detection of coronary artery disease (CAD) as it allows the visualization of the luminal stenosis and the vessel wall simultaneously. Detecting the presence of coronary atherosclerosis and stenosis alone does not provide sufficient prognostic information on future events. It has been shown that plaques with high risk features, such as positive remodeling, low attenuation, napkin ring sign, and spotty calcification, are associated with subsequent coronary events. ${ }^{1}$ Moreover, recent studies suggested that plaques with high risk features have a higher likelihood to cause ischemia independent of the degree of stenosis. Ischemia-guided revascularization compared to revascularization based on stenosis severity shows prognostic benefit. ${ }^{2-4}$ Therefore, to define the functional significance of atherosclerotic lesions, it is of utmost importance to be able to provide appropriate conservative or invasive therapy.

Invasive fractional flow reserve (FFR) is the gold standard technique to detect lesion-specific ischemia. However, due to the technical advances that cardiac CT underwent during the past decade, noninvasive ischemia assessment has become feasible. Isotropic resolution and high-quality image datasets allow for advanced functional assessments and computational simulations.

With the application of computational fluid dynamic modeling to cardiac CT datasets flow simulation and pressure estimation became feasible allowing the calculation of FFR values noninvasively. The simulation does not require any extra image acquisition or special medication, which eases the implementation of FFR-CT into the daily routine. All major studies aiming to assess the diagnostic performance of FFR-CT have been conducted with HeartFlow FFRCT (HeartFlow Inc, Redwood City, California, USA), which is a vendor-independent application. ${ }^{5}$ The main disadvantage of HeartFlow FFRCT however, is that it only provides off-site analysis, which takes $12-24$ hours and requires data transfer. Other vendors have developed on-site FFR simulation algorithms; however, these still have to be validated.

Transluminal attenuation gradient (TAG) quantifies the contrast material attenuation change along the vessel, which refers to flow circumstances within coronaries. ${ }^{6}$ TAG shows a consistent decrease and marks maximal stenosis with a significant attenuation drop. This technique can be applied on single heart beat-acquired CTA images, and similarly to the FFR-CT application, the regular CTA protocols do not need to be extended. Additionally, with the implementation of corrected contrast opacification, the inhomogeneous attenuation can be adjusted for in datasets acquired in more than one step. Initial validation studies confirmed diagnostic improvement if TAG was combined with conventional coronary CTA as compared to CTA only. ${ }^{7}$ However, probably due to the differences in scanners, reconstruction techniques, and contrast material injection protocols, there is currently no uniform cutoff value for TAG.

It is of high clinical importance to understand the association of ischemia with the presence of high-risk plaque features. In two articles published in two consecutive issues of JCE, Orzan et al. demonstrated that TAG shows a good correlation with invasive FFR values. Furthermore, they showed that increased TAG values are associated with high-risk plaque characteristics, which 
suggest the potential of this technique to help in the quest to identify vulnerable lesions. ${ }^{8,9}$ We appreciate Orzan et al. for the first steps to bring us closer to understand the relationship of TAG and high-risk plaque features. Larger studies are warranted to further elucidate the intricate relationship of lesion-specific ischemia and plaque vulnerability.

\section{CONFLICT OF INTEREST}

Nothing to declare.

\section{ACKNOWLEDGEMENT}

Julia Karady MD received funding from the HungarianAmerican Fulbright Commission.

\section{REFERENCES}

1. Maurovich-Horvat P, Ferencik M, Voros S, Merkely B, Hoffmann U. Comprehensive plaque assessment by coronary CT angiography. Nat Rev Cardiol. 2014;11:390-402. doi: 10.1038/nrcardio.2014.60.

2. Kim YH, Ahn JM, Park DW, et al. Impact of ischemia-guided revascularization with myocardial perfusion imaging for patients with multivessel coronary disease. J Am Coll Cardiol. 2012;60:181-190. doi: 10.1016/j.jacc.2012.02.061.

3. Pijls NH, Fearon WF, Tonino PA, et al. Fractional flow reserve versus angiography for guiding percutaneous coronary intervention in patients with multivessel coronary artery disease: 2-year follow-up of the FAME (Fractional Flow Reserve Versus Angiography for Multivessel Evaluation) study. J Am Coll Cardiol. 2010;56:177-184. doi: 10.1016/j. jacc.2010.04.012.

4. Tonino PA, Fearon WF, De Bruyne B, et al. Angiographic versus functional severity of coronary artery stenoses in the FAME study fractional flow reserve versus angiography in multivessel evaluation. J Am Coll Cardiol. 2010;55:2816-21. doi: 10.1016/j.jacc.2009.11.096.

5. Koo BK, Erglis A, Doh JH, et al. Diagnosis of ischemia-causing coronary stenoses by noninvasive fractional flow reserve computed from coronary computed tomographic angiograms. Results from the prospective multicenter DISCOVER-FLOW (Diagnosis of Ischemia-Causing Stenoses Obtained Via Noninvasive Fractional Flow Reserve) study. J Am Coll Cardiol. 2011;58:1989-1997. doi: 10.1016/j.jacc.2011.06.066.

6. Steigner ML, Mitsouras D, Whitmore AG, et al. Iodinated contrast opacification gradients in normal coronary arteries imaged with prospectively ECG-gated single heart beat 320-detector row computed tomography. Circ Cardiovasc Imaging. 2010;3:179-186. doi: 10.1161/ CIRCIMAGING.109.854307.

7. Choi JH, Min JK, Labounty TM, et al. Intracoronary transluminal attenuation gradient in coronary CT angiography for determining coronary artery stenosis. JACC Cardiovasc Imaging. 2011;4:1149-57. doi: 10.1016/j.jcmg.2011.09.006.

8. Orzan M, Chițu M. A Comparative Preliminary Study on CT Contrast Attenuation Gradient Versus Invasive FFR in Patients with Unstable Angina. Journal of Cardiovascular Emergencies. 2017;3:72-78. doi: 10.1515/jce-2017-0012.

9. Orzan M, Hodas R, Dobra M, Rat N, Chitu M, Benedek I. Transluminal Contrast Attenuation Gradient Is Associated with Coronary Plaque Vulnerability - a Computed Tomography Angiography-based Study. Journal of Cardiovascular Emergencies. 2017;3:121-127. doi: 10.1515/jce-2017-0016. 